\title{
Identities of the plactic monoid
}

\author{
Łukasz Kubat • Jan Okniński
}

Received: 12 September 2013 / Accepted: 30 April 2014 / Published online: 10 June 2014

(C) The Author(s) 2014. This article is published with open access at Springerlink.com

\begin{abstract}
It is shown that the plactic monoid $M$ of rank 3 satisfies the identity $w v v w v w=w v w v v w$ where $v=x y y x x y x y y x$ and $w=x y y x y x x y y x$. This is accomplished by first showing that certain simple monoids related to $M$ satisfy this identity. These simple monoids are natural generalizations of the bicyclic monoid $B$, which satisfies the identity $w=v$ by a result of Adjan.
\end{abstract}

Keywords Plactic monoid · Identity · Bicyclic monoid

\section{Introduction}

For an integer $n \geq 1$ we consider the finitely presented monoid $M_{n}=\left\langle a_{1}, \ldots, a_{n}\right\rangle$ defined by the relations

$$
\begin{array}{ll}
a_{i} a_{k} a_{j}=a_{k} a_{i} a_{j} & \text { for } i \leq j<k, \\
a_{j} a_{i} a_{k}=a_{j} a_{k} a_{i} & \text { for } i<j \leq k .
\end{array}
$$

It is called the plactic monoid of rank $n$ and was introduced in [14]. It is known that the elements of $M_{n}$ can be written in a canonical form, which implies that they are in a

Communicated by Mohan Putcha.

\section{Ł. Kubat $(\varangle)$}

Institute of Mathematics, Polish Academy of Sciences,

Sniadeckich 8, 00-956 Warsaw, Poland

e-mail: kubat.lukasz@gmail.com

\section{J. Okniński}

Institute of Mathematics, University of Warsaw,

Banacha 2, 02-097 Warsaw, Poland

e-mail: okninski@mimuw.edu.pl 
one-to-one correspondence with Young tableaux of certain type. In particular, $M_{n}$ has polynomial growth of degree $n(n+1) / 2$. Because of its strong connections to Young tableaux, the plactic monoid has proved to be a very powerful tool in representation theory and in algebraic combinatorics, see [7,13]. The combinatorics of $M_{n}$ has been extensively studied, recently including also the aspect of the Gröbner-Shirshov bases $[2,6,11]$. The algebraic structure and representations of the monoid algebra $K\left[M_{n}\right]$ of $M_{n}$ over a field $K$ have been investigated, $[4,10]$. This paper is motivated by the following conjecture.

\section{Conjecture The plactic monoid of any rank $n \geq 1$ satisfies a nontrivial identity.}

On one hand, a motivation comes from general problems concerning existence of identities in classes of finitely generated semigroups of polynomial growth. In particular, it is known (by results of Gromov and Grigorchuk) that a finitely generated cancellative semigroup of polynomial growth satisfies a nontrivial identity, see [15]. However, as shown by Shneerson in [17], this is no longer true without the cancellativity hypothesis. On the other hand, linear semigroups of polynomial growth satisfy a nontrivial identity, [15], Corollary 5.8, while there exist linear semigroups satisfying a nontrivial identity that have subexponential growth, see [15], Example on page 186, and [5] for further results in this direction. Further connections between polynomial growth and the theory of varieties of semigroups were explored in [18], motivated in particular by Sapir's problem on relatively free semigroups of polynomial growth [19]. While simple monoids seem to be of special interest from the point of view of varieties of semigroups, it is also worth mentioning that the structure of $M_{n}$ heavily depends on certain simple monoids discovered in [10]. These monoids can be considered as generalizations of the bicyclic monoid $B$. Therefore, a result of Adjan [1], asserting that the bicyclic monoid $B=\langle p, q \mid q p=1\rangle$ satisfies the identity

$$
x y y x x y x y y x=\operatorname{xy} y x y x x y y x .
$$

is the starting point for our approach.

On the other hand, the conjecture is motivated by the fact that a class of strongly related monoids, called Chinese monoids $C_{n}, n \geq 1$, satisfies identity (1). This class was introduced and studied in [3]. Actually, the monoids $C_{n}$ and $M_{n}$ are isomorphic if $n \leq 2$. It was shown in [9] that $C_{n}$ embeds into the product $B^{i} \times \mathbb{Z}^{j}$, for some positive integers $i, j$ depending on $n$, where $\mathbb{Z}$ denotes the additive group of integers. This is a consequence of the description of the minimal prime spectrum of the semigroup algebra $K\left[C_{n}\right]$ over a field $K$. Therefore, since the bicyclic monoid satisfies identity (1), $C_{n}$ also satisfies this identity.

Applying some of the results of [10] we prove in this paper that $M=M_{3}$ satisfies the identity

$$
w v v w v w=w v w v v w
$$

where $v=v(x, y)=x y y x x y x y y x$ and $w=w(x, y)=x y y x y x x y y x$. Our approach is based on homomorphic images of $M$ arising from natural maps $K[M] \rightarrow K[M] / P$, where $P$ are minimal prime ideals of $K[M]$ and on certain infinite dimensional linear representations of $M$. All such primes $P$ of $K[M]$ and all irreducible representations 
of $M$ have been determined [10], while in case of plactic monoids $M_{n}$ with $n \geq 4$ the corresponding problems remain a challenge. In particular, there is no obvious starting point for an attempt to extend our proof to the class of all plactic monoids. One might expect that for every $n \geq 4$ the monoid $M_{n}$ satisfies some identities, which however would be more complicated than those satisfied by $M_{n-1}$ (unlike the case of Chinese monoids mentioned above) and thus the proofs might be more involved, accordingly.

\section{Identities of $M_{3}$}

Adjan's result was recently reproved in a completely different but quite complicated way in [8], starting from an embedding of $B$ into the semigroup of $2 \times 2$ tropical matrices. We start with a simple conceptual proof of this result, and for a more detailed study of identities satisfied by the bicyclic monoid we refer to [16]. It is worth mentioning that one of the key ideas of the proof of our main result is based on a strategy similar to the argument given below.

Proposition 2.1 The bicyclic monoid B satisfies the identity

$$
w(x, y)=v(x, y)
$$

Proof We apply the faithful representation $\phi: B \rightarrow \operatorname{End}(V)$, where $V$ is a vector space over a field $K$ with a basis $\left\{e_{s}: s \geq 0\right\}$ and

$$
\phi(p)\left(e_{s}\right)=e_{s+1}, \quad \phi(q)\left(e_{s}\right)= \begin{cases}0 & \text { if } s=0 \\ e_{s-1} & \text { if } s>0\end{cases}
$$

see Exercise 11.9 in [12]. For simplicity we write $b e_{s}=\phi(b)\left(e_{s}\right)$ for $b \in B$. Notice that $p^{i} q^{j}\left(e_{s}\right)=e_{s-j+i}$ if it is nonzero, which holds exactly when $j \leq s$. In particular, if $b e_{s}=0$ then $b e_{t}=0$ for every $t \leq s$.

Let $x=p^{i} q^{j}, y=p^{k} q^{m} \in B$. In order to show that $w(x, y)=v(x, y)$ we may assume that $i+k-j-m \geq 0$, because otherwise we apply the involution on $B$ defined by $p \mapsto q, q \mapsto p$, which reduces the problem to this case. It is now enough to show that for every $s \geq 0$ we have $x y x y y x e_{s}=$ yxxyyxe . We may assume that one of these elements is nonzero. Then $x y y x e_{s} \neq 0$. Write $y x e_{s}=e_{s^{\prime}}, x y y x e_{s}=e_{s^{\prime \prime}}$. Then

$$
s^{\prime \prime}=s^{\prime}+(i+k-j-m) \geq s^{\prime}=s+(i+k-j-m) \geq s
$$

by the assumption.

Since $s^{\prime \prime} \geq s$ and $y x e_{s} \neq 0$, we must have $y x x y y x e_{s}=y x e_{s^{\prime \prime}} \neq 0$. Second, since $s^{\prime \prime} \geq s^{\prime}$ and $x y e_{s^{\prime}}=e_{s^{\prime \prime}} \neq 0$, we also have $x y x y y x e_{s}=x y e_{s^{\prime \prime}} \neq 0$.

Hence, both elements $w e_{s}, v e_{s}$ are nonzero, and therefore they are both equal to $e_{s+3(i+k-j-m)}$. The result follows. 
It is known that $a_{n} a_{n-1} \cdots a_{2} a_{1}$ is a central and regular element of $K\left[M_{n}\right]$. In particular $M_{2}=\langle a, b\rangle \subseteq M_{2}\langle b a\rangle^{-1} \cong B \times \mathbb{Z}$ and $M_{2}$ satisfies identity (1) by Proposition 2.1, as was already noticed in [9].

We recall from [10] basic information on certain homomorphic images $N_{1}$ and $N_{2}$ of $M=M_{3}$ that were used in the context of the classification of minimal prime ideals of the monoid algebra $K[M]$. Namely, $M$ is described by the presentation

$$
M=\langle a, b, c\rangle,
$$

where

$$
\begin{aligned}
& a b a=b a a, \quad b a b=b b a, \quad a c a=c a a, \quad c a c=c c a \\
& c b b=b c b, \quad c b c=c c b, \quad b a c=b c a, \quad a c b=c a b
\end{aligned}
$$

and we define $N_{1}=M /(a c=c a)$ and $N_{2}=M /(b a c b=c b b a)$. Here, for any elements $u_{1}, u_{2} \in M$ by $M /\left(u_{1}=u_{2}\right)$ we mean the factor semigroup $M / \tau$, where $\tau$ is the congruence on $M$ generated by the pair $\left(u_{1}, u_{2}\right)$. We will denote the generators of $M$ and their natural images in the considered homomorphic images of $M$ in the same way, if unambiguous.

The element $z=c b a$ is a central and regular element of $K[M]$ and also of $K\left[N_{i}\right]$ for $i=1,2$, and one can consider the central localizations $M\langle z\rangle^{-1}$ and $N_{i}\langle z\rangle^{-1}$. Moreover we have

$$
\begin{aligned}
M\langle z\rangle^{-1} \cong M /(z=1) \times\left\langle z, z^{-1}\right\rangle \cong M /(z=1) \times \mathbb{Z} \\
N_{i}\langle z\rangle^{-1} \cong N_{i} /(z=1) \times\left\langle z, z^{-1}\right\rangle \cong N_{i} /(z=1) \times \mathbb{Z}
\end{aligned}
$$

and

$$
N_{i}\langle z\rangle^{-1} /(z=1) \cong N_{i} /(z=1) .
$$

The map $f: M \rightarrow M$ defined by $a \mapsto c b, b \mapsto c a, c \mapsto b a$ is an antimonomorphism, which induces an involution $M /(z=1) \rightarrow M /(z=1)$. Moreover, $M$ is a subdirect product of $N_{1}$ and $N_{2}$ and $M /(z=1)$ is a subdirect product of $N_{1} /(z=1)$ and $N_{2} /(z=1)$. Notice that $f$ induces an antiisomorphim of the latter two monoids. It follows that in order to decide whether $M$ satisfies nontrivial identities it is sufficient to verify this for the monoid $N_{1} /(z=1)$. Namely, let us say that a pair of words $s=s(x, y), t=t(x, y)$ in $x, y$ is reversive if one obtains the same two words (as a set) when reading $s(x, y), t(x, y)$ backwards. Then we get

Lemma 2.2 The monoids $M, M /(c b a=1)$ and $N_{1} /(c b a=1)$ satisfy the same identities of the form $s(x, y)=t(x, y)$, where $s, t$ is a reversive pair of words.

Every element $u \in N_{1}$ can be uniquely written in the form

$$
u=(c b a)^{k_{1}}(b a)^{k_{2}} a^{k_{3}}(c b)^{k_{4}} b^{k_{5}} c^{k_{6}},
$$

where $k_{i} \geq 0$, see [10], Lemma 2.3. Hence, in view of (5), every element $\bar{u}$ of the monoid $N_{1} /(z=1)$ can be uniquely written in the form 


$$
\bar{u}=(b a)^{k_{2}} a^{k_{3}}(c b)^{k_{4}} b^{k_{5}} c^{k_{6}}
$$

where $k_{i} \geq 0$. And it follows that $N_{1} /(z=1)$ is a simple monoid, and hence so is $N_{2} /(z=1)$. Since $c b \cdot a=c \cdot b a$ is the unity of $N_{1} /(z=1)$, these monoids carry some flavor of the monoid $B$, see [10], Proposition 2.6.

Consider the natural homomorphisms

$$
\phi_{1}: N_{1} \rightarrow N_{1} /(a b=b a)
$$

and

$$
\phi_{2}: N_{1} \rightarrow N_{1} /(b c=c b)
$$

So, the former makes the image of $a$ central. It is easy to see that

$$
N_{1} /(a b=b a) \cong\langle a\rangle \times\langle b, c\rangle \cong \mathbb{N} \times M_{2}
$$

and similarly

$$
N_{1} /(b c=c b) \cong\langle c\rangle \times\langle a, b\rangle \cong \mathbb{N} \times M_{2},
$$

with $\mathbb{N}$ denoting the additive monoid of non-negative integers.

Assume that two elements

$$
\begin{aligned}
\alpha & =(c b a)^{k_{1}}(b a)^{k_{2}} a^{k_{3}}(c b)^{k_{4}} b^{k_{5}} c^{k_{6}}, \\
\alpha^{\prime} & =(c b a)^{k_{1}^{\prime}}(b a)^{k_{2}^{\prime}} a^{k_{3}^{\prime}}(c b)^{k_{4}^{\prime}} b^{k_{5}^{\prime}} c^{k_{6}^{\prime}}
\end{aligned}
$$

of $N_{1}$ satisfy $\phi_{i}(\alpha)=\phi_{i}\left(\alpha^{\prime}\right)$ for $i=1,2$. Using the canonical form of elements of $\mathbb{N} \times M_{2}$ it is easy to see that

$$
\phi_{1}(\alpha)=a^{k_{1}+k_{2}+k_{3}}(c b)^{k_{1}+k_{4}} b^{k_{2}+k_{5}} c^{k_{6}}, \quad \phi_{2}(\alpha)=c^{k_{1}+k_{4}+k_{6}}(b a)^{k_{1}+k_{2}} a^{k_{3}} b^{k_{4}+k_{5}}
$$

and similar presentations hold for the elements $\phi_{1}\left(\alpha^{\prime}\right)$ and $\phi_{2}\left(\alpha^{\prime}\right)$. Consequently, we get

$$
k_{1}+k_{2}+k_{3}=k_{1}^{\prime}+k_{2}^{\prime}+k_{3}^{\prime}, k_{1}+k_{4}=k_{1}^{\prime}+k_{4}^{\prime}, k_{2}+k_{5}=k_{2}^{\prime}+k_{5}^{\prime}, k_{6}=k_{6}^{\prime}
$$

and

$$
k_{1}+k_{4}+k_{6}=k_{1}^{\prime}+k_{4}^{\prime}+k_{6}^{\prime}, k_{1}+k_{2}=k_{1}^{\prime}+k_{2}^{\prime}, k_{4}+k_{5}=k_{4}^{\prime}+k_{5}^{\prime}, k_{3}=k_{3}^{\prime} .
$$

This is equivalent to the conditions

$$
k_{1}+k_{2}=k_{1}^{\prime}+k_{2}^{\prime}, k_{1}+k_{4}=k_{1}^{\prime}+k_{4}^{\prime}, k_{2}+k_{5}=k_{2}^{\prime}+k_{5}^{\prime}, k_{3}=k_{3}^{\prime}, k_{6}=k_{6}^{\prime} .
$$

If $k_{1}=k_{1}^{\prime}$ then clearly $k_{q}=k_{q}^{\prime}$ for $q=1,2,3,4,5,6$, so that $\alpha=\alpha^{\prime}$. Hence, assume for example that $k_{1}>k_{1}^{\prime}$. Let $k_{1}^{\prime}=i$ and $k_{1}=i+s$ for some $s \geq 1$. Then also 
$k_{2}^{\prime}=k_{2}+s, k_{4}^{\prime}=k_{4}+s, k_{5}=k_{5}^{\prime}+s$ and $k_{2}^{\prime}=k_{2}+s$. Put $j=k_{2}, k=k_{3}, l=$ $k_{4}, m=k_{5}, r=k_{6}$. Then $\alpha, \alpha^{\prime}$ are of the form

$$
\begin{aligned}
\alpha & =(c b a)^{i+s}(b a)^{j} a^{k}(c b)^{l} b^{m+s} c^{r}=\left[(c b a)^{i+s}(b a)^{j} a^{k}\right] b^{s}\left[(c b)^{l} b^{m} c^{r}\right], \\
\alpha^{\prime} & =(c b a)^{i}(b a)^{j+s} a^{k}(c b)^{l+s} b^{m} c^{r}=\left[(c b a)^{i}(b a)^{j} a^{k}\right]\left((b a)^{s}(c b)^{s}\right)\left[(c b)^{l} b^{m} c^{r}\right]
\end{aligned}
$$

and the images of $\alpha, \alpha^{\prime}$ in $N_{1} /(z=1)$ are of the form

$$
(b a)^{j} a^{k} b^{s}(c b)^{l} b^{m} c^{r}, \quad(b a)^{j} a^{k}\left((b a)^{s}(c b)^{s}\right)(c b)^{l} b^{m} c^{r},
$$

respectively. So $\alpha, \alpha^{\prime}$ are in the congruence $\rho$ on $N_{1}$ generated by all pairs $\left((c b a)^{s} b^{s},(b a)^{s}(c b)^{s}\right), s \geq 1$. It follows that $\rho=\operatorname{Ker} \phi_{1} \cap \operatorname{Ker} \phi_{2}$, the intersection of the congruences determined by $\phi_{1}$ and $\phi_{2}$ and $N_{1} / \rho$ embeds into the monoid $N_{1} /(a b=b a) \times N_{1} /(b c=c b)$.

We know that $\mathbb{N} \times M_{2}$ satisfies Adjan's identity (1) because the plactic monoid of rank 2 satisfies every identity holding in the bicyclic monoid. Hence, in view of (8) and (9), $N_{1} / \rho$ satisfies this identity.

In particular, for every $x, y \in N_{1}$ the elements $x y^{2} x x y x y^{2} x, x y^{2} x y x x y^{2} x$ must be of the form described in (10) and (11), while their images in $N_{1} /(z=1)$ are of the form (12). Moreover, if $k_{1}=k_{1}^{\prime}$ (equivalently $s=0$ ) then $\alpha=\alpha^{\prime}$, with the notation as above.

Hence we obtain the following consequence.

Lemma 2.3 Let $w_{1}, w_{2}$ be two distinct words in the free monoid of rank two of the same total degree. Assume also that every evaluation of the words $w_{1}(v, w)$ and $w_{2}(v, w)$ in $N_{1}$ (with $v=v(x, y), w=w(x, y)$ defined in (2)) leads to two elements of $N_{1}$ with equal exponents of cba in the canonical form. Then $N_{1}$ satisfies a nontrivial identity, namely

$$
w_{1}\left(x y^{2} x x y x y^{2} x, x y^{2} x y x x y^{2} x\right)=w_{2}\left(x y^{2} x x y x y^{2} x, x y^{2} x y x x y^{2} x\right) .
$$

For our main result we will use an idea analogous to that used in the proof of Proposition 2.1. Consider the representation $\phi$ of $M$ coming from Proposition 3.6 in [10] (with $\beta=\gamma=1$ ). Namely, let $V$ be a vector space over a field $K$ with basis $\left\{e_{p q}: p, q \geq 0\right\}$. Let the action of $a, b, c \in M$ on $V$ be given by

$a e_{p q}=e_{p, q+1}, \quad b e_{p q}=\left\{\begin{array}{ll}e_{p q} & \text { if } q=0, \\ e_{p+1, q-1} & \text { if } q>0,\end{array} \quad c e_{p q}= \begin{cases}0 & \text { if } p=0 \\ e_{p-1, q} & \text { if } p>0\end{cases}\right.$

Then this action makes $V$ a (simple) left $K[M]$-module. Actually, this is also a $K\left[N_{1}\right]$ module and a $K\left[N_{1} /(z=1)\right]$-module.

We will use the fact that

$$
b^{s} e_{p q}=e_{p+\min \{s, q\}, q-\min \{s, q\}}
$$


and another easy consequence of the definition

$$
(b a)^{s}(c b)^{s} e_{p q}= \begin{cases}0 & \text { if } p+q<s, \\ e_{p+\min \{s, q\}, q-\min \{s, q\}} & \text { if } p+q \geq s .\end{cases}
$$

In particular, $b^{s} e_{p q}=(b a)^{s}(c b)^{s} e_{p q}$ if $(b a)^{s}(c b)^{s} e_{p q} \neq 0$. Moreover, the latter is 0 only if $p+q<s$ and then $b^{s} e_{p q}=e_{p+q, 0}$.

Lemma 2.4 The representation $\phi$ is faithful on $N_{1} /(z=1)$.

Proof We use the canonical form (7) of elements in $N_{1} /(z=1)$. Suppose that $\phi(t)=$ $\phi\left(t^{\prime}\right)$ for some

$$
t=(b a)^{k_{2}} a^{k_{3}}(c b)^{k_{4}} b^{k_{5}} c^{k_{6}}, \quad t^{\prime}=(b a)^{k_{2}^{\prime}} a^{k_{3}^{\prime}}(c b)^{k_{4}^{\prime}} b^{k_{5}^{\prime}} c^{k_{6}^{\prime}} \in N_{1} /(z=1),
$$

where $k_{i} \geq 0$. If $k_{6}^{\prime}>k_{6}$ then $t^{\prime} e_{k_{6}, k_{4}}=0$ and $t e_{k_{6}, k_{4}} \neq 0$. So we may assume $k_{6}^{\prime}=k_{6}$. If $k_{4}^{\prime}>k_{4}$ then $t^{\prime} e_{k_{4}+k_{6,0}}=0$ and $t e_{k_{4}+k_{6,0}} \neq 0$. So $k_{4}=k_{4}^{\prime}$. Now $t e_{p q}=e_{p-k_{6}+k_{5}+k_{2}, q-k_{5}-k_{4}+k_{3}}$ if $p \geq k_{6}$ and $q \geq k_{4}+k_{5}$. Hence $k_{5}+k_{2}=k_{5}^{\prime}+k_{2}^{\prime}$ and $-k_{5}+k_{3}=-k_{5}^{\prime}+k_{3}^{\prime}$. If $k_{5}^{\prime} \geq k_{5}$ then $t^{\prime} e_{k_{6}, k_{4}+k_{5}^{\prime}}=e_{k_{5}^{\prime}+k_{2}, k_{3}}$ and $t e_{k_{6}, k_{4}+k_{5}^{\prime}}=e_{k_{5}+k_{2}, k_{3}}$. Hence $k_{5}^{\prime}=k_{5}$ and the assertion follows easily.

We will often use the following standard fact [10,14].

Lemma 2.5 If $t \in M$ then $t=c u b u^{\prime}$ a for some $u, u^{\prime} \in M$ if and only if $t=$ cbat $^{\prime}$ for some $t^{\prime} \in M$.

We will also use the involution $\epsilon: M \rightarrow M$ determined by $\epsilon(a)=c, \epsilon(b)=b$, $\epsilon(c)=a$. It also leads to an involution of $N_{1}$, also denoted by $\epsilon$ for simplicity.

Theorem 2.6 The monoid $M=M_{3}$ satisfies the identity $w v v w v w=w v w v v w$, where $v=x y y x x y x y y x$ and $w=x y y x y x x y y x$ represent the left-hand and respectively the right-hand side of identity (1).

Proof The main difficulty of the proof is to show that this identity is satisfied on $N_{1}$. Our approach will be based on Lemma 2.4 and on Lemma 2.3. For $x, y \in N_{1}$ and $w=w(x, y), v=v(x, y)$ we have

$$
\{w, v\}=\left\{(c b a)^{i+s}(b a)^{j} a^{k} b^{m} b^{s}(c b)^{l} c^{r},(c b a)^{i}(b a)^{j} a^{k} b^{m}\left((b a)^{s}(c b)^{s}\right)(c b)^{l} c^{r}\right\}
$$

for some $s$, see (10),(11). Hence, in order to decide whether $\alpha(w(x, y), v(x, y))=$ $\beta(w(x, y), v(x, y))$ in $N_{1}$ for some $x, y \in N_{1}$ and some words $\alpha, \beta$ of the same degree in each of the two variables, it is enough to show that $\alpha(w(x, y), v(x, y))$ and $\beta(w(x, y), v(x, y))$ have the same kernel on the basis $\left\{e_{p q}: p, q \geq 0\right\}$ of the module $V$ introduced before Lemma 2.4. (Indeed, in this case their images must be equal in $N_{1} /(z=1)$ by the observation made before Lemma 2.4 , so that a degree argument shows that they are equal in $N_{1}$.) We will use this observation several times without further comment. 
First, we consider the case where

$$
\begin{aligned}
w & =(c b a)^{i+s}(b a)^{j} a^{k} b^{m} b^{s}(c b)^{l} c^{r}, \\
v & =(c b a)^{i}(b a)^{j} a^{k} b^{m}\left((b a)^{s}(c b)^{s}\right)(c b)^{l} c^{r} .
\end{aligned}
$$

We will show that in $N_{1}$ either $w v w v=v w w v$ and $v w v w=w v v w$, or $w v w v=$ $w v v w$ and $v w v w=v w w v$. The case where the roles of $w$ and $v$ are switched then also follows because of the symmetry of these equalities.

We write $u=(b a)^{j} a^{k}, t=(c b)^{l} b^{m} c^{r}$, so that

$$
w=(c b a)^{i+s} u b^{s} t, \quad v=(c b a)^{i} u\left((b a)^{s}(c b)^{s}\right) t
$$

and consider the following four cases:

1. $r \leq j$ and $l \leq k$,

2. $r \leq j$ and $l>k$,

3. $r>j$ and $l \leq k$,

4. $r>j$ and $l>k$.

It is easy to see that the product $t u$ is divisible by $(c b a)^{r+l},(c b a)^{r+k+\min \{l-k, j-r\}}$, $(c b a)^{j+l},(c b a)^{k+j}$ in these cases, respectively. So, the central regular element $c b a$ appears in this way the same number of times in each of the elements $w v w v, v w w v, v w v w$ and $w v v w$. Moreover, the initial segment $u$ of each of these elements and the terminal segment $t$ do not affect the exponent of $c b a$ in the canonical form of these elements in $N_{1}$. Therefore, deleting from $u$ and from $t$ the appropriate factors (those that produce the maximal power of $c b a$ in the product $t u$ ) we may assume that $u, t$ have one of the following forms (corresponding to the cases $1-4$ listed above):

1. $u=(b a)^{j}(a)^{k}$ and $t=(b)^{m}$,

2. $u=(b a)^{j}$ and $t=(c b)^{l}(b)^{m}$,

3. $u=(a)^{k}$ and $t=(b)^{m}(c)^{r}$,

4. $u=1$ and $t=(c b)^{l}(b)^{m}(c)^{r}$.

In the second case, if the exponents of $b a$ and $c b$ are nonzero, the situation is also reduced to case 1 . or case 4 ., due to the possible cancellation of $c b a$ coming from the factor $c b$ ba arising from $t u$.

So, cancelling the appropriate exponents of $c b a$ in $w$ and $v$, we may assume that the elements $w, v$ are of one of the following forms (corresponding to cases 1, 4, and 3 , respectively):

(i) $w=(b a)^{j} a^{k} b^{m+s}$ and $v=a^{k}(b a)^{j+s}(c b)^{s} b^{m}$,

(ii) $w=b^{m+s}(c b)^{l} c^{r}$ and $v=(b a)^{s}(c b)^{l+s} b^{m} c^{r}$,

(iii) $w=a^{k} b^{m+s} c^{r}$ and $v=a^{k}(b a)^{s}(c b)^{s} b^{m} c^{r}$.

In the following computations we often apply Lemma 2.5 and the relations defining $N_{1}$ without further comment.

We consider the three cases indicated above. 
Case (i). The proof of this case will be based on Lemma 2.4. First, we will show that $v w v w=w v v w$. As observed in the first paragraph of the proof, to establish the latter equality it is enough to check that

$$
v w v w e_{p q} \neq 0 \text { iff } w v v w e_{p q} \neq 0 \text { for all } p, q \geq 0 \text {. }
$$

Before we proceed to the proof of (15) let us first note that $w e_{p q} \neq 0$ for all $p, q \geq 0$. Moreover $v e_{p q}=0$ if and only if $(c b)^{s} b^{m} e_{p q}=0$. Since we have

$$
(c b)^{s} b^{m} e_{p q}= \begin{cases}0, & \text { if } p+q<s, \\ e_{p-s+\min \{q, m+s\}, q-\min \{q, m+s\},} & \text { if } p+q \geq s,\end{cases}
$$

it is easy to see that $v e_{p q}=0$ if and only if $p+q<s$. Next, let us note that elements $b a$ and $a$ acting on $e_{p q}$ increase $p+q$ exactly by 1 . Similarly, $c b$ annihilates $e_{p q}$ or decreases $p+q$ exactly by 1 , while $b$ does not change $p+q$. Hence we conclude that if $e_{p^{\prime} q^{\prime}}=w e_{p q}$ or $e_{p^{\prime} q^{\prime}}=v e_{p q} \neq 0$, then necessarily $p+q \leq p^{\prime}+q^{\prime}$.

In order to prove (15) we may assume that one of the elements $v w v w e_{p q}, w v v w e_{p q}$ is nonzero. In this situation, we have $v w e_{p q} \neq 0$. Therefore, $e_{p_{1} q_{1}}=w e_{p q} \neq 0$, $e_{p_{2} q_{2}}=v e_{p_{1} q_{1}} \neq 0$ and $e_{p_{3} q_{3}}=w e_{p_{2} q_{2}} \neq 0$, for some natural numbers $p_{1}, p_{2}, p_{3}, q_{1}, q_{2}, q_{3}$. As observed above, in this case $p_{1}+q_{1} \leq p_{2}+q_{2} \leq p_{3}+q_{3}$. If $v e_{p_{2} q_{2}}=0$, then using $p_{1}+q_{1} \leq p_{2}+q_{2}<s$ we would get $v e_{p_{1} q_{1}}=0$, a contradiction. Hence $e_{p_{3}^{\prime} q_{3}^{\prime}}=v e_{p_{2} q_{2}} \neq 0$ for some $p_{3}^{\prime}, q_{3}^{\prime}$. Finally, from $p_{2}+q_{2} \leq p_{3}+q_{3}$ we conclude that $v e_{p_{3} q_{3}} \neq 0$ and consequently we get

$$
\begin{aligned}
& v_{w v w e_{p q}}=v w v e_{p_{1} q_{1}}=v w e_{p_{2} q_{2}}=v e_{p_{3} q_{3}} \neq 0 \\
& w_{v v w e_{p q}}=w_{v v e_{p_{1} q_{1}}}=w v e_{p_{2} q_{2}}=w e_{p_{3}^{\prime} q_{3}^{\prime}} \neq 0 .
\end{aligned}
$$

Hence, by (15), in Case (i) the elements $v w v w$ and $w v v w$ are equal in $N_{1}$. In a similar way one shows that $w v w v e_{p q} \neq 0$ if and only if $v w w v e_{p q} \neq 0$ for all $p, q \geq 0$ and hence $w v w v=v w w v$.

Case (ii). In this case we apply the involution $\epsilon$ of $N_{1}$ and we get

$$
\begin{aligned}
\epsilon(w) & =\epsilon\left(b^{m+s}(c b)^{l} c^{r}\right)=a^{r}(b a)^{l} b^{m+s}, \\
\epsilon(v) & =\epsilon\left((b a)^{s}(c b)^{l+s} b^{m} c^{r}\right)=a^{r} b^{m}(b a)^{l+s}(c b)^{s} .
\end{aligned}
$$

This leads to Case (i). Therefore, we get relations: $v w v w=v w w v$ and $w v w v=$ wvvw in $N_{1}$.

Case (iii). First, assume that $r \geq k$. We consider three subcases:

(I) $s \leq k \leq r$,

(II) $k \leq s \leq r$,

(III) $k \leq r \leq s$.

If (I) holds then

$$
w v=\left[a^{k} b^{m+s} c^{r}\right]\left[a^{k}(b a)^{s}(c b)^{s} b^{m} c^{r}\right]=(c b a)^{s} u
$$


for some $u \notin c b a M$ and

$$
v w=\left[a^{k}(b a)^{s}(c b)^{s} b^{m} c^{r}\right]\left[a^{k} b^{m+s} c^{r}\right]=(c b a)^{s} u^{\prime}
$$

for some $u^{\prime} \notin c b a M$. By Lemma 2.3 we get $w v=v w$ in $N_{1}$.

If (II) holds then

$$
\begin{aligned}
w v & =\left[a^{k} b^{m+s} c^{r}\right]\left[a^{k}(b a)^{s}(c b)^{s} b^{m} c^{r}\right] \\
& =a^{k} b^{m+s} c^{r-s} a^{k}(c b a)^{s}(c b)^{s} b^{m} c^{r} \\
& =(c b a)^{s} a^{k}(b a)^{k} b^{m+s-k} c^{r-s}(c b)^{s} b^{m} c^{r} \\
& =(c b a)^{s} a^{k}(b a)^{k} t
\end{aligned}
$$

where $t \in\langle b, c\rangle$. Then

$$
v(w v)=u(c b)^{s} c^{r}(c b a)^{s} a^{k}(b a)^{k} t=u(c b a)^{s+k+k} t_{1}
$$

where $u \in\langle a, b\rangle, t_{1} \in\langle b, c\rangle$. So $v w v \notin(c b a)^{s+2 k+1} M$. Moreover

$$
\begin{aligned}
v w & =\left[a^{k}(b a)^{s}(c b)^{s} b^{m} c^{r}\right]\left[a^{k} b^{m+s} c^{r}\right] \\
& =a^{k}(b a)^{s}(c b a)^{k} b^{m}(c b)^{s-k} c^{r} b^{m+s} c^{r} \\
& =(c b a)^{k} a^{k}(b a)^{s} t^{\prime}
\end{aligned}
$$

where $t^{\prime} \in\langle b, c\rangle$. Hence

$$
v(v w)=u(c b)^{s} c^{r}(c b a)^{k} a^{k}(b a)^{s} t^{\prime}=u(c b a)^{s+k+k} t_{2}
$$

where $t_{2} \in\langle b, c\rangle$. So $v v w \notin(c b a)^{s+2 k+1} M$.

By Lemma 2.3 we get $v w v=v v w$ in $N_{1}$.

Similarly

$$
w(w v)=\left[a^{k} b^{m+s} c^{r}\right]\left[(c b a)^{s} a^{k}(b a)^{k} t\right] \in(c b a)^{s+k} M \backslash(c b a)^{s+k+1} M
$$

and

$$
w(v w)=\left[a^{k} b^{m+s} c^{r}\right]\left[(c b a)^{k} a^{k}(b a)^{s} t^{\prime}\right] \in(c b a)^{s+k} M \backslash(c b a)^{s+k+1} M .
$$

Hence we also have $w w v=w v w$. So in Case (II) we also get $w v w v=w v v w$ and $v w w v=v w v w$ in $N_{1}$.

If (III) holds then

$$
\begin{aligned}
w v & =\left[a^{k} b^{m+s} c^{r}\right]\left[a^{k}(b a)^{s}(c b)^{s} b^{m} c^{r}\right] \\
& =a^{k} b^{m+s}(c b a)^{r}(b a)^{s-r} a^{k}(c b)^{s} b^{m} c^{r} \\
& =(c b a)^{r} a^{k} b^{2 m+s-k}(b a)^{s-r+k}(c b)^{s} c^{r}
\end{aligned}
$$




$$
\begin{aligned}
v w & =\left[a^{k}(b a)^{s}(c b)^{s} b^{m} c^{r}\right]\left[a^{k} b^{m+s} c^{r}\right] \\
& =a^{k}(b a)^{s} b^{m}(c b a)^{k}(c b)^{s-k} c^{r} b^{m+s} c^{r} \\
& =(c b a)^{k} a^{k}(b a)^{s} b^{m}(c b)^{s-k+r} b^{m+s-r} c^{r} \\
& =(c b a)^{k} a^{k}(b a)^{s} b^{2 m+s-r}(c b)^{s-k+r} c^{r} .
\end{aligned}
$$

Then

$$
\begin{aligned}
w v v w & =\left[(c b a)^{r} a^{k} b^{2 m+s-k}(b a)^{s-r+k}(c b)^{s} c^{r}\right]\left[(c b a)^{k} a^{k}(b a)^{s} b^{2 m+s-r}(c b)^{s-k+r} c^{r}\right] \\
& =(c b a)^{k+r} u(c b)^{s} c^{r} a^{k}(b a)^{s} t \\
& =(c b a)^{k+r} u(c b a)^{k+r}(c b)^{s-k}(b a)^{s-r} t \\
& =(c b a)^{2 k+s+r} u^{\prime} t^{\prime}
\end{aligned}
$$

where $u, u^{\prime} \in\langle a, b\rangle, t, t^{\prime} \in\langle b, c\rangle$ and

$$
\begin{aligned}
w v w v & =\left[(c b a)^{r} a^{k} b^{2 m+s-k}(b a)^{s-r+k}(c b)^{s} c^{r}\right]\left[(c b a)^{r} a^{k} b^{2 m+s-k}(b a)^{s-r+k}(c b)^{s} c^{r}\right] \\
& =(c b a)^{r+r} u^{\prime}(c b)^{s} c^{r} a^{k}(b a)^{s-r+k} t^{\prime \prime} \\
& =(c b a)^{r+r} u^{\prime}(c b a)^{k}(c b)^{s-k} c^{r}(b a)^{s-r+k} t^{\prime \prime} \\
& =(c b a)^{s+r+2 k} u^{\prime} t^{\prime \prime \prime}
\end{aligned}
$$

where $u^{\prime} \in\langle a, b\rangle, t^{\prime \prime}, t^{\prime \prime \prime} \in\langle b, c\rangle$. By Lemma 2.3 we get $w v w v=w v v w$ in $N_{1}$.

Moreover

$$
\begin{aligned}
v w w v & =\left[(c b a)^{k} a^{k}(b a)^{s} b^{2 m+s-r}(c b)^{s-k+r} c^{r}\right]\left[(c b a)^{r} a^{k} b^{2 m+s-k}(b a)^{s-r+k}(c b)^{s} c^{r}\right] \\
& =u(c b a)^{k+r}(c b)^{s-k+r} c^{r} a^{k}(b a)^{s-r+k} t \\
& =u(c b a)^{k+r+s-r+k} c^{r} b^{s-r+k}(c b)^{2 r-2 k} a^{k} t \\
& =u(c b a)^{2 k+s+k} t^{\prime}
\end{aligned}
$$

where $u \in\langle a, b\rangle, t, t^{\prime} \in\langle b, c\rangle$ (because the degree of $b$ in $(c b)^{2 r-2 k} b^{s-r+k}$ is $s+r-$ $k \geq k$ ). Also

$$
\begin{aligned}
v w v w & =\left[(c b a)^{k} a^{k}(b a)^{s} b^{2 m+s-r}(c b)^{s-k+r} c^{r}\right]\left[(c b a)^{k} a^{k}(b a)^{s} b^{2 m+s-r}(c b)^{s-k+r} c^{r}\right] \\
& =u^{\prime}(c b a)^{k+k}(c b)^{s-k+r} c^{r} a^{k}(b a)^{s} t^{\prime \prime} \\
& =u^{\prime}(c b a)^{k+k}(c b)^{s-k+r} a^{k}(c b a)^{r}(b a)^{s-r} t^{\prime \prime} \\
& =u^{\prime}(c b a)^{2 k+s+k} t^{\prime \prime \prime}
\end{aligned}
$$

where $u^{\prime} \in\langle a, b\rangle, t^{\prime \prime}, t^{\prime \prime \prime} \in\langle b, c\rangle$ (because the degree of $a$ in $a^{k}(b a)^{s-r}$ is $s+k-r \leq$ $s-k+r)$.

By Lemma 2.3 we get $v w w v=v w v w$ in $N_{1}$. 
It remains to consider the situation where $r<k$ in Case (iii). Then applying the involution $\epsilon$ on $N_{1}$ we get

$$
\epsilon(v)=(a)^{r} b^{m}(b a)^{s}(c b)^{s} c^{k}, \quad \epsilon(w)=a^{r} b^{m+s} c^{k} .
$$

Therefore, this reduces the situation to the case where $r>k$, considered above. This means that $v w w v=w v w v$ and $w v v w=v w v w$ in $N_{1}$.

Conclusion: if $w=w(x, y)$ and $v=v(x, y)$ for some $x, y \in N_{1}$ then either $w v w v=v w w v$ and $v w v w=w v v w$ or $w v w v=w v v w$ and $v w v w=v w w v$. Hence in the former case we have

$$
w v(v w v w)=w v(w v v w)
$$

while in the latter

$$
(w v v w) v w=(w v w v) v w .
$$

Thus, the identity $w v v w v w=w v w v v w$ is satisfied in $N_{1}$, as claimed at the beginning of the proof.

Recall that $v$ (viewed as a word in $x, y$ ) is obtained by reading backwards the word $w$. Therefore, an argument as that leading to Lemma 2.2 shows that also in $N_{2}$ we get that either $w v w v=v w w v$ and $v w v w=w v v w$, or $w v w v=w v v w$ and $v w v w=v w w v$. So $N_{2}$ also satisfies the identity $w v v w v w=w v w v v w$. Since $M$ is a subdirect product of $N_{1}$ and $N_{2}$, this identity is also satisfied in $M$.

One can ask whether $M$ satisfies a simpler identity. We conclude by showing that $M$ does not satisfy Adjan's identity $w(x, y)=v(x, y)$. Let $x=a^{3} c$ and $y=(b a) b(c b)^{2}$. Then

$$
x y=(c b a) a^{3} b(c b)^{2}, \quad y x=(c b a)^{2}(b a)^{2} c
$$

and

$$
x y y x=(c b a) a^{3} b(c b)^{2}(c b a)^{2}(b a)^{2} c=(c b a)^{5} a^{3} b^{3} c
$$

so that it is easy to see that

$$
\begin{aligned}
w(x, y) & =(c b a)^{5} a^{3} b^{3} c(c b a) a^{3} b(c b)^{2}(c b a)^{5} a^{3} b^{3} c \\
& =(c b a)^{11} a^{3} b^{3} a^{3}(c b)^{3} a^{3} b^{3} c \\
& =(c b a)^{14} a^{3}(b a)^{3} b^{3} c \\
v(x, y) & =(c b a)^{5} a^{3} b^{3} c(c b a)^{2}(b a)^{2} c(c b a)^{5} a^{3} b^{3} c \\
& =(c b a)^{12} a^{3} b^{3} c(b a)^{2} c a^{3} b^{3} c \\
& =(c b a)^{13} a^{3}(b a) b^{3} c a^{3} b^{3} c \\
& =(c b a)^{13} a^{3}(b a)^{4} b^{2}(c b) c
\end{aligned}
$$


are written in the canonical forms in $M$, whence they are different. Notice that $v w$ and $w v$ again have a different exponent of $c b a$ in their canonical forms, namely 29 and 28, respectively; whence they are different. But we get $w v v w=v w v w$ and $v w w v=w v w v$.

Acknowledgments Research supported by NCN Grant 2011/03/N/ST1/00108 and MNiSW Grant N201 420539 (Poland).

Open Access This article is distributed under the terms of the Creative Commons Attribution License which permits any use, distribution, and reproduction in any medium, provided the original author(s) and the source are credited.

\section{References}

1. Adjan, S.I.: Defining relations and algorithmic problems for groups and semigroups. In: Proceedings of Steklov Institute of Mathematics, vol. 85. American Mathematical Society, Providence (1966)

2. Cain, A.J., Gray, R., Malheiro, A.: Finite Gröbner-Shirshov bases for Plactic algebras and biautomatic structures for Plactic monoids. arXiv:1205.4885v2

3. Cassaigne, J., Espie, M., Krob, D., Novelli, J.-C., Hivert, F.: The Chinese monoid. Int. J. Algebra Comput. 11(3), 301-334 (2001)

4. Cedó, F., Okniński, J.: Plactic algebras. J. Algebra 274, 97-117 (2004)

5. Cedó, F., Okniński, J.: Semigroups of matrices of intermediate growth. Adv. Math. 212, 669-691 (2007)

6. Chen, Y., Li, J.: New approach to Schensted-Knuth normal forms. arXiv:1106.4753v1

7. Fulton, W.: Young Tableaux, London Mathematical Society Student Texts, vol. 35. Cambridge University Press, New York (1997)

8. Izhakian, Z., Margolis, S.W.: Semigroup identities in the monoid of two-by-two tropical matrices. Semigr. Forum 80, 191-218 (2010)

9. Jaszuńska, J., Okniński, J.: Structure of Chinese algebras. J. Algebra 346, 31-81 (2011)

10. Kubat, Ł., Okniński, J.: Plactic algebra of rank 3. Semigr. Forum 84, 241-266 (2012)

11. Kubat, Ł, Okniński J.: Gröbner-Shirshov bases for plactic algebras. Algebra Colloquium (to appear)

12. Lam, T.Y.: Exercises in Clasical Ring Theory. Springer, New York (2003)

13. Lascoux, A., Leclerc, B., Thibon, J.-Y.: The plactic monoid. In: Algebraic Combinatorics on Words, pp. 164-196. Cambridge University Press, Cambridge (2002)

14. Lascoux, A., Schützenberger, M.P.: Le monoide plaxique. In: Noncommutative Structures in Algebra and Geometric Combinatorics (Naples, 1978), vol. 109 of Quad. "Ricerca Sci.", pp. 129-156. CNR, Rome (1981)

15. Okniński, J.: Semigroups of Matrices. World Scientific, Singapore (1998)

16. Pastijn, F.: Polyhedral convex cones and the equational theory of the bicyclic semigroup. J. Aust. Math. Soc. 81, 63-96 (2006)

17. Shneerson, L.M.: Identities in finitely generated semigroups of polynomial growth. J. Algebra 154, 67-85 (1993)

18. Shneerson, L.M.: Polynomial growth in semigroup varieties. J. Algebra 320, 2218-2279 (2008)

19. Shevrin, L.N. (ed.): The Sverdlovsk Notebook. Unsolved Problems of the Theory of Semigroups (Russian), 3rd ed. Ural State University, Sverdlovsk (1989) 